\title{
TUNISIE, DES MIGRANTS SUBSAHARIENS TOUJOURS EXCLUS DU RÊVE DÉMOCRATIQUE
}

\author{
Vincent Geisser
}

Centre d'information et d'études sur les migrations internationales | «igrations Société »

2019/3 $\mathrm{N}^{\circ} 177$ | pages 3 à 18

ISSN 0995-7367

Article disponible en ligne à l'adresse :

https://www.cairn.inforevue-migrations-societe-2019-3-page-3.htm

Distribution électronique Cairn.info pour Centre d'information et d'études sur les migrations internationales.

(C) Centre d'information et d'études sur les migrations internationales. Tous droits réservés pour tous pays.

La reproduction ou représentation de cet article, notamment par photocopie, n'est autorisée que dans les limites des conditions générales d'utilisation du site ou, le cas échéant, des conditions générales de la licence souscrite par votre établissement. Toute autre reproduction ou représentation, en tout ou partie, sous quelque forme et de quelque manière que ce soit, est interdite sauf accord préalable et écrit de l'éditeur, en dehors des cas prévus par la législation en vigueur en France. Il est précisé que son stockage dans une base de données est également interdit. 


\title{
Tunisie, des migrants subsahariens toujours exclus du rêve démocratique
}

\author{
Vincent GEISSER
}

«Ce n'est pas parce que l'Occident et l'Europe abandonnent les migrants qu'on doit faire la même chose! ».

Chamseddine Bourassine, pêcheur tunisien de la ville portuaire de Zarzis ${ }^{\mathrm{I}}$

"Cette situation est indigne de la Tunisie postrévolution. Assurer un accueil décent aux immigrés subsahariens est un devoir dicté par la nouvelle constitution tunisienne qui consacre les droits de l'homme».

Saïd Ben Sedrine, économiste tunisien ${ }^{2}$

En cet automne 2019, la Tunisie connaît deux grands événements politiques censés consacrés son statut symbolique d'unique «rescapé des printemps arabes »: une élection présidentielle anticipée à la miseptembre, suite au décès du président de la République Béji Caïd Essebsi, et une élection législative début octobre, toutes les deux au suffrage universel direct, dans un contexte de relatif respect du choix des électeurs, contrastant ainsi avec les manipulations électorales du régime autoritaire de Zine El Abidine Ben Ali. Néanmoins, derrière cette façade de l'exceptionnalisme démocratique «à la tunisienne ${ }^{3}$ pointent des pratiques moins avouables comme la collusion entre les milieux d'affaires et les acteurs politiques, la corruption généralisée, le contrôle des médias par des groupes de pression, les tentatives d'achat des suffrages des citoyens et l'instrumentalisation de l'appareil judiciaire et sécuritaire à des fins politico-électorales, contribuant à ternir l'image idyllique de la «jeune démocratie tunisienne», pourtant récompensée par un prix Nobel de la paix en décembre 2015. Mais ne nous y trompons pas : il s'agit moins d'une restauration de la dictature ou d'un retour de l'ancien régime que d'une démocratisation profondément ambivalente, consacrant des pratiques hybrides entre autoritarisme et démocratie, une sorte de «zone

1. MZALOUAT, Haïfa, "Les pêcheurs tunisiens, nouvelle cible de I'Italie" [En ligne], Inkyfada, 10 septembre 2018, https://inkyfada.com/fr/2018/10/09/pecheurs-tunisie-passeurs-italie/.

2. BEN SEDRINE, Saïd, Défis à relever pour un accueil décent de la migration subsaharienne en Tunisie, La Marsa : Fondation Friedrich Ebert, décembre 2018, 88 p. (voir pp.7-8).

3. CAMAU, Michel, L'exception tunisienne. Variation sur un mythe, Paris : IRMC/Karthala, 2018, $254 \mathrm{p}$. 
grise » qui affecte autant les élites politiques et économiques que les simples citoyens et qui nous fait dire que «la Tunisie est une jeune démocratie avec les maux d'une ancienne démocratie $\gg{ }^{4}$. De ce point de vue, la gestion publique des migrations et de l'exil constitue une entrée pertinente pour saisir les limites, les contradictions et les ambiguités du processus de démocratisation en Tunisie, caractérisé par des tendances de rupture par rapport au passé dictatorial (1956-20II), mais aussi des éléments de continuité, voire de consolidation, des logiques de contrôle social, de verrouillage sécuritaire de l'espace public, de violences institutionnelles à l'égard des populations vulnérables et de production systémique de discriminations sociales, territoriales, sexuelles et raciales. En somme, «dis-moi comment tu traites tes minorités, tes immigrés et tes réfugiés, je te dirai quel est l'état de ta démocratie!».

Il est vrai qu'avant la révolution de 20II, la Tunisie était considérée par ses partenaires européens - notamment la France et l'Italie - et les bailleurs de fonds (Banque mondiale et Fonds monétaire international), comme le «bon élève » en matière de gestion migratoire et de lutte contre le terrorisme, les deux registres étant intimement liés dans la nouvelle doxa sécuritaire : les migrants irréguliers sont perçus comme des menaces potentielles pour l'ordre public des pays de destination car susceptibles d'alimenter les réseaux du terrorisme islamique. De ce fait, les réformes de la politique migratoire adoptées par le régime de Ben Ali cherchaient principalement à répondre aux injonctions européennes, positionnant le pays comme un sous-traitant sécuritaire et un vigile de l'Europe, en espérant ainsi en récolter les dividendes en termes de légitimité internationale ${ }^{5}$. Autrement dit, renforcer sa crédibilité gestionnaire pour mieux masquer en interne les atteintes aux libertés et aux droits de l'homme : "en instaurant la loi du 3 février 2004, Ben Ali entendait répondre aux exigences européennes de contrôle de ses frontières. Cette loi sur les passeports et les documents de voyage avait été d'abord conçue pour poursuivre les passeurs, mais a fini par être appliquée par les juges pour les migrants euxmêmes et toute personne qui leur viendrait en aide $»^{6}$. La thématique de la «bonne gouvernance » des flux migratoires représentait alors une rhétorique euphémisée pour asseoir les bases d'une coopération sécuritaire entre les pays de départ, les pays de transit et les pays d'accueil, écartant délibérément les citoyens ordinaires, les organisations non gouvernemen-

4. "Dimanche, les Tunisiens iront voter sans conviction... Ou pas du tout. Entretien avec Vincent Geisser" [En ligne], La Montagne.fr, 12 septembre 2019, https://www.lamon tagne.fr/paris-75000/actualites/dimanche-les-tunisiens-iront-voter-sans-conviction-oupas-du-tout_13639308/.

5. GEISSER, Vincent, "Des Guantanamo aux portes de l'Europe? L'externalisation au secours de nos 'amis' les dictateurs", Migrations Société, vol. 21, n 122, mars-avril 2009, pp. 3-10.

6. JANNE D'OTHÉE, Nathalie, "Tunisie. Huit ans après la Révolution, une politique migratoire inchangée" [En ligne], cncd.be, 15 janvier 2019, https://www.cncd.be/Tunisie-Huit-ansapres-la. 
tales (ONG) humanitaires, les associations de solidarité et même les parlements nationaux des processus décisionnels, comme si les politiques migratoires relevaient d'un « domaine à part », non soumis aux procédures de contrôle démocratique. L'une des décisions illustrant le mieux cette prédominance du paradigme sécuritaire en matière migratoire est la conclusion d'accords de réadmission entre la Tunisie et l'Italie, violant toutes les conventions internationales en matière de protection des droits des migrants et des réfugiés, comme le rappelle l'universitaire Jean-Pierre Cassarino, fin observateur de la politique migratoire tunisienne depuis plus de vingt ans: "L'entente entre la Tunisie et l'Italie, datée du 28 janvier 2009, reflète parfaitement l'incidence de cette hiérarchie [de priorités] où l'opérabilité l'emporte sur le respect des normes internationales en matière de respect des droits humains, dont ceux, notamment, des réfugiés et demandeurs d'asile. Enfin, une autre fonction de la hiérarchie de priorités, une fois consolidée dans les mentalités et les discours officiels, consiste à justifier le recours inéluctable au mal nécessaire, donc à rendre acceptable l'inacceptable au moyen d'affirmations plausibles et dépourvues de justification $»^{7}$.

Toutefois, la Tunisie de Ben Ali n'était pas uniquement un acteur passif et une simple exécutante des politiques migratoires européennes. Cette vision, parfois véhiculée par certains milieux militants, tend à déresponsabiliser les gouvernants des pays en voie de développement. Or, le régime autoritaire tunisien jouait aussi un rôle actif dans la sécurisation des migrations, selon un processus de localisation qui lui permettait de renforcer son contrôle policier sur la société, et plus particulièrement sur la jeunesse. En ce sens, la politique migratoire pratiquée sous la dictature de Ben Ali constituait un terrain d'expérimentation et d'application des politiques sécuritaires qui s'exerçaient sur l'ensemble de la société, et notamment à l'égard des populations perçues comme turbulentes, c'est-à-dire les couches sociales les plus vulnérables habitant les territoires les plus défavorisés du Nord-Ouest, du Centre et du Sud : "la lutte contre les migrations dites "illégales" a permis au régime de dissimuler les véritables causes des migrations en provenance de Tunisie et de faire taire les exclus du "miracle économique" tunisien. Ces derniers étaient généralement décrits dans les médias comme des individus attirés par le rêve de l'Eldorado européen. Cette vision paternaliste et infantilisante, reprise à l'envi en Europe, permettait de détourner l'attention du public des motifs réels de départ des migrants, à savoir le sous-emploi, la pauvreté, le mécontentement social et la violence politique $»^{8}$. En somme, la politique de coopération migratoire « imposée » par l’Europe aux pays du Maghreb en général, et à la Tunisie en particulier, confortait les régimes locaux dans leur orientation autoritaire et paternaliste dans la gestion de leur propre société, avec la

7. CASSARINO, Jean-Pierre, "Le gouvernement des migrations en Tunisie : vers un nouveau paradigme ?", in : ALLAL, Amin ; GEISSER, Vincent (sous la direction de), Tunisie : une démocratisation au-dessus de tout soupçon? Paris : CNRS Éditions, 2018, pp. 295-309 (voir p. 303).

8. Ibidem (voir pp. 298-299). 
caution des États dits démocratiques qui ont volontairement fermé les yeux sur les atteintes récurrentes aux droits de l'homme et aux libertés fondamentales qui touchaient autant les migrants que les citoyens nationaux. Ceci explique très largement qu'à l'exception du $\operatorname{Maroc}^{9}$ — les militants des droits de l'homme marocains ont critiqué dès les années I990 le paradigme sécuritaire - les débats sur les migrations étaient totalement tabous dans l'espace public des pays du Maghreb, les informations sur le sujet étant généralement contrôlées et censurées par le ministère de l'Intérieur ${ }^{\mathrm{IO}}$.

Sur ce plan, il s'est bien produit un « effet révolution », libérant la parole publique sur les migrations : longtemps occultée et réprimée, la question migratoire a connu consécutivement aux «printemps arabes » un processus de "détabouisation », se traduisant par des formes de réappropriation citoyenne et militante de la critique du paradigme sécuritaire. Limité autrefois à quelques cercles d'initiés, aux ONG ou aux professionnels des politiques publiques, le débat sur les migrations et l'accueil des réfugiés s'est diffusé, après 20II, à de larges secteurs de la société tunisienne, suscitant des prises de position, des manifestations sur la voie publique, mais aussi des mobilisations de solidarité organisées par des collectifs citoyens, des associations humanitaires et des syndicats de salariés : «Sur le plan intérieur tunisien, la révolution et le changement politique majeur qui en a résulté, notamment après les élections du 23 octobre 20II, ont permis pour la première fois de placer les débats autour de la migration sur la scène publique et dans les médias, d'ouvrir la voie aux organisations de la société civile pour contribuer à ce débat et même de peser sur l'action des institutions et des organismes gouvernementaux dans le domaine des migrations $\gg{ }^{\mathrm{II}}$. Une organisation comme le Forum tunisien pour les droits économiques et sociaux (FTDES), créé en 2OII et disposant de sections locales dans la plupart des villes du pays (Tunis, Kairouan, Kasserine, Monastir, Gafsa, etc.), a joué un rôle majeur dans la dénonciation des politiques sécuritaires de gestion de la migration. Sa contribution au débat n'a pas seulement consisté à critiquer la prédominance du paradigme sécuritaire, mais aussi à faire le lien entre la question démocratique et la question migratoire, en ne limitant pas la réflexion aux harragas (les « brûleurs de frontières $»^{\mathrm{I}^{2}}$ ) mais en l'étendant aussi aux autres catégories de migrants, notamment les immigrés et les réfugiés subsaha-

9. FELIU MARTíNEZ, Laura, "Les migrations en transit au Maroc. Attitudes et comportement de la société civile face au phénomène", L'Année du Maghreb, V, 2009, pp. 343-362.

10. Dans le cas de la Tunisie, le débat était porté depuis la France par une organisation comme la Fédération tunisienne pour une citoyenneté des deux rives (FTCR) dont les communiqués étaient la plupart du temps censurés en Tunisie. Le site internet de la FTCR faisait régulièrement l'objet d'attaques par les services sécuritaires tunisiens.

11. BOUBAKRI, Hassan, "Les migrations en Tunisie après la révolution", Confluences Méditerranée, vol. 4, $n^{\circ}$ 87, 2013, pp. 31-46 (voir p. 32).

12. SOUIAH, Farida, "'Brûler' les frontières : fuite ou contestation", in : ALLAL, Amin ; GEISSER, Vincent (sous la direction de), Tunisie : une démocratisation au-dessus de tout soupçon? op. cit., pp. 377-393. 
riens transitant ou résidant dans le pays. Le FTDES a porté la question migratoire dans l'arène politique et constitutionnelle, en la traitant comme une priorité de la construction démocratique tunisienne : pas de démocratie solide et durable sans traitement digne des émigrés et des immigrés ${ }^{13}$. Il est vrai que, dans les premiers mois qui ont suivi la révolution, ces mobilisations associatives ont été encouragées par les nouvelles élites gouvernantes issues des rangs de la résistance à la dictature, qui entendaient promouvoir une conception plus équilibrée des relations Nord/Sud et remettre en cause le prisme exclusivement sécuritaire des politiques migratoires, qui représentait pour elles un "héritage honteux » de l'ancien régime. Dès lors, la réforme de la politique migratoire s'est inscrite dans le cadre global de la démocratisation des institutions et de la volonté gouvernementale de redonner de la dignité aux citoyens tunisiens, y compris ceux résidant à l'étrange (diaspora) I $^{14}$ : "c'est dans un contexte chargé de tensions et de revendications sociales, de mobilisations multiformes et de crises politiques et institutionnelles qu'un nouveau prisme est apparu aux yeux des acteurs dès les premiers mois de 20II. Les référents de l'ancien régime portant sur la gestion des migrations, aussi bien légales qu'irrégulières, ont rapidement été remis en question, à l'instar de l'AGMI ${ }^{15}$ qui, à une échelle plus globale, les avait légitimés ${ }^{16}$. Cependant, comme nous le verrons ultérieurement, cette coalition entre associations de solidarité, militants des droits de l'homme et nouvelles élites gouvernantes n'a été qu'éphémère, ces dernières ne résistant pas au retour en force d'une conception sécuritaire de la gestion des migrations pour cause de «bonnes relations » avec les bailleurs de fonds et avec les États européens, mais aussi en raison du nombre croissant d'attaques terroristes sur le territoire tunisien. Les idéaux utopiques et tiers-mondistes des premiers gouvernements postrévolutionnaires ont progressivement cédé la place à une vision "réaliste », froide et pragmatique, renouant avec le paradigme sécuritaire d'avant $201 \mathrm{I}$ : «Le traitement de la mobilité des personnes et des migrations est devenu plus complexe avec l'émergence du terrorisme et des menaces aux frontières, surtout terrestres, de la Tunisie. [...] La mobilité des Tunisiens et des étrangers ne se pose plus uniquement en termes de contrôles aux

13. Sur le rôle du FTDES dans la publicisation du débat sur l'émigration/immigration, voir BOUBAKRI, Hassan, "Migration et asile en Tunisie depuis 2011 : vers de nouvelles figures migratoires ?", Revue européenne des migrations internationales, vol. 31, n 3-4, 2015, pp. 17-39 ; BEN SEDRINE, Saïd, Défis à relever pour un accueil décent de la migration subsaharienne en Tunisie, op. cit.

14. GEISSER, Vincent ; LIMAM, Wajdi, "L'an prochain à Tunis? Les binationaux franco-tunisiens au prisme des héritages militants et des subjectivités diasporiques", in : ALLAL, Amin : GEISSER, Vincent (sous la direction de), Tunisie : une démocratisation au-dessus de tout soupçon?, op. cit., pp. 413-432.

15. «Agenda pour la gestion des migrations internationales», qui désigne la coopération bilatérale ou multilatérale entre les États et les organisations supranationales sur les questions migratoires.

16. CASSARINO, Jean-Pierre, "Le gouvernement des migrations en Tunisie : vers un nouveau paradigme?", op. cit. (voir p. 305). 
frontières et de maitrise des flux, mais aussi en termes des risques que représentent ces passages au niveau sécuritaire, ce qui ouvre la voie au retour du traitement sécuritaire, voire même militariste, des flux migratoires ${ }^{17}$.

Parmi les acteurs collectifs tunisiens qui ont contribué à la publicisation et à la détabouisation du débat sur la question migratoire après la révolution, il convient de citer également l'Union générale tunisienne du travail (UGTT), syndicat historique (fondé en 1946 par Ferhat Hached ${ }^{18}$ ), auréolé par sa participation à la chute de la dictature : l'UGTT sera d'ailleurs l'une des principales initiatrices du Réseau syndical migrations méditerranéennes subsahariennes (RSMMS) : «en août 20I4, I7 organisations syndicales du Maghreb (Maroc, Algérie, Tunisie, Mauritanie et Égypte), du nord de la Méditerranée (Italie, France) et de l'Afrique subsaharienne (Côte-d'Ivoire, Mali, Niger et Sénégal), ont créé le Réseau syndical migrations méditerranéennes subsahariennes (RSMMS) ; c'est le fruit d'une longue réflexion entamée en 2009 à Hammamet en Tunisie qui s'est prolongée à Dakar au Sénégal en 20I3, puis la même année à Turin en Italie. La déclaration de Casablanca est l'acte de naissance de ce réseau. Elle traduit la volonté de ses membres de coordonner leurs efforts en vue de protéger les travailleurs migrants tout au long de leurs déplacements, prévenir la dégradation de leurs conditions de vie et de travail et lutter contre toute forme de discrimination, de racisme et de xénophobie $»^{19}$. Dans le même temps, certaines fédérations sectorielles et régionales de l'UGTT ont parfois tenu un discours plus ambivalent à l'égard des migrants subsahariens, ces derniers étant indirectement accusés de faire baisser le coût du travail et de développer une course folle à la productivité, menaçant le statut et les conditions de travail des salariés tunisiens. Mais d'une manière générale, l'UGTT a joué un rôle majeur pour dénoncer la reconduction du paradigme sécuritaire dans les politiques migratoires, et la soumission de l'État tunisien aux injonctions de l'Union européenne ${ }^{20}$.

Au-delà des ONG internationales, des associations locales et des syndicats, ce sont aussi des milliers de citoyens ordinaires et des acteurs anonymes qui se sont mobilisés après 201 en faveur des migrants et des réfugiés subsahariens. Les habitants des zones frontalières ont souvent

17. BOUBAKRI, Hassan, "Migration et asile en Tunisie depuis 2011: vers de nouvelles figures migratoires?", art. cité (voir p. 35)

18. Leader syndical toujours populaire en Tunisie, assassiné par des colons français en 1952. ॥ est considéré comme l'un des martyrs de la lutte de libération nationale.

19. BEN SEDRINE, Saïd, Défis à relever pour un accueil décent de la migration subsaharienne en Tunisie, op. cit. (voir p. 8).

20. En mai 2018, I'UGTT a ouvert ses premiers « espaces migrants » à Tunis, Sousse et Sfax, afin d'offrir «aux travailleurs migrants, quel que soit leur statut, l'accès à des informations fiables (légis/ation régissant les relations employeurs/employés, information sur les services de base, etc.), à des formations spécifiques (éducation financière, mécanismes de protection en cas d'abus, etc.) et à des services concrets (conciliation en cas de conflits de travail) ». Passage cité sur le site de l'Organisation internationale du Travail : https://www.ilo. org/global/topics/labour-migration/events-training/WCMS_629992/lang--fr/index.htm. 
apporté les premiers secours aux individus et aux familles fuyant la guerre fratricide en Libye. En dépit d'un racisme anti-noir aux racines historiques profondes dans la société tunisienne ${ }^{21}$, les gestes de solidarité n'ont pas été rares : ce sont souvent de simples citoyens de condition sociale modeste qui sont venus compenser les défaillances du HautCommissariat des Nations unies pour les réfugiés (HCR) et des pouvoirs publics tunisiens en matière d'accueil, organisant des formes d'hospitalité totalement désintéressées. Ces mobilisations spontanées ont également été initiées par des jeunes diplômés tunisiens des centres urbains, qui ont traversé tout le pays afin de mettre en ouvre de nombreuses actions bénévoles pour venir en aide aux migrants en général, et aux Subsahariens en particulier. Sur ce plan, l'effervescence démocratique des premiers mois de la révolution s'est aussi traduite par une ouverture à l'«altérité » et une volonté de combattre les réflexes de peur et les comportements xénophobes à l'égard de l'« étranger africain » et du « minoritaire noir » qui avaient libre cours sous le régime autoritaire de Ben $\mathrm{Ali}^{22}$.

Ce sont aussi les professions directement affectées dans leurs activités par les «drames humains » de la migration (noyades en mer, naufrages des embarcations, agressions physiques par les gardes-côtes et les milices privées, etc.). Par exemple, les pêcheurs ont été conduits à déployer ces dernières années des actions de solidarité en direction des migrants, au risque de subir eux-mêmes des représailles policières et des sanctions pénales de la part de l'État tunisien ou des autorités européennes. Ainsi, ce sont des centaines de pêcheurs tunisiens qui, de Kélibia (pointe de la péninsule du cap Bon) aux ports du Golfe de Gabès, en passant par Mahdia et les îles de Djerba et Kerkennah, se sont improvisés quotidiennement sauveteurs volontaires pour secourir les migrants sur des embarcations de fortune ou inhumer dignement des corps sans vie rejetés par la mer, à l'instar de ce cimetière des "migrants anonymes », aménagé à l'initiative des pêcheurs de la ville côtière de Zarzis. Sans être forcément politisés ou syndiqués, ces pêcheurs tunisiens constituent aujourd'hui la profession la plus engagée dans les actions humanitaires en direction des migrants, impliqués dans les campagnes de dénonciation des «effets meurtriers» de la gestion sécuritaire du phénomène migratoire ${ }^{23}$. D'aucuns n'hésitent plus à critiquer ouvertement l'hypocrisie des politi-

21. ABDELHAMID, Maha, "De la libération de la parole raciste à l'émergence d'un mouvement contre le racisme anti-noir", in : ALLAL, Amin ; GEISSER, Vincent (sous la direction de), Tunisie : une démocratisation au-dessus de tout soupçon?, op. cit., pp. 343-356.

22. POUESSEL, Stéphanie, "De Tunis à Tozeur, descente vers le Sud. Retour à un paradigme 'africain' au Maghreb", IBLA, vol. 75, n²10, 2012, pp. 297-327.

23. Sur les actions de solidarité des pêcheurs tunisiens à l'égard des migrants, voir LEBEL, Audrey, "L'homme qui donne une sépulture aux migrants" [En ligne], Regards.fr, 14 juin 2019, http://www.regards.fr/monde/article/I-homme-qui-donne-une-sepulture-aux-mi grants ; MZALOUAT, Haïfa, "Les pêcheurs tunisiens, nouvelle cible de I'Italie" [En ligne], Inkyfada.com, 10 septembre 2018, https://inkyfada.com/fr/2018/10/09/pecheurs-tuni sie-passeurs-italie/. 
ques migratoires et à remettre en cause la lâcheté inhumaine de leur propre gouvernement et des États européens ${ }^{24}$ : «On m'accuse de sauver des vies ? Dans ce cas, je suis fier de cette accusation ${ }^{25}$, déclarait ainsi Chamseddine Bourassine, pêcheur de Zarzis, interpellé et incarcéré par les autorités italiennes.

Toutefois, il convient de ne pas verser dans une représentation victimaire et misérabiliste selon laquelle les migrants subsahariens subiraient passivement les situations de domination sociale, d'exploitation économique et de discrimination raciale. Depuis janvier 20II, profitant du nouveau contexte de liberté associative, des organisations de migrants africains se sont créées à Tunis et dans les principales villes du pays. Elles développent de nombreuses initiatives publiques pour alerter l'opinion tunisienne, les ONG, les médias et les responsables politiques sur leur condition de migrants africains en Tunisie. Il est vrai que jusqu'à présent, ces mobilisations subsahariennes tendent à se limiter aux étudiants africains qui disposent d'un capital scolaire et culturel suffisamment élevé pour porter leur «cause » dans l'espace public mais qui n'en sont pas moins confrontés à des formes de précarité professionnelle, d'exploitation économique, de violence physique et/ou symbolique qui se manifestent régulièrement à leur égard dans la société tunisienne ${ }^{26}$. Parmi les organisations subsahariennes les plus actives, citons l'association des étudiants et stagiaires africains en Tunisie (AESAT), dont l'objectif est à la fois de créer un réseau de solidarité, de venir en aide aux étudiants subsahariens (dont la plupart travaillent parallèlement dans le secteur informel pour financer leurs études) et d'interpeller les autorités tunisiennes sur les discriminations et le racisme qu'ils subissent quotidiennement ${ }^{27}$.

Huit ans après la révolution, ces mobilisations inédites de la «société civile » tunisienne ont-elles porté leurs fruits ? La libération de la parole sur la question migratoire a-t-elle débouché sur des initiatives législatives et constitutionnelles ? Le «paradigme sécuritaire » de la période Ben Ali a-t-il été remis en cause ou continue-t-il à structurer les politiques migratoires et, au-delà, les attitudes et les comportements des pouvoirs publics tunisiens à l'égard des migrants et des réfugiés subsahariens ? Les réponses à ces questions sont nécessairement contrastées, venant nuancer très

24. GEISSER, Vincent, "Méditerranée, 'morte nostrum': un terrorisme de l'indifférence?", Migrations Société, vol. 27, $\mathrm{n}^{\circ}$ 159-160, mai-août 2015, pp. 3-12.

25. Cité par LOUARN, Anne-Diandra, "Tous les pêcheurs de Zarzis ont déjà secouru des migrants en mer et on continuera" [En ligne], Infomigrants.net, 24 septembre 2018, https://www.infomigrants.net/fr/post/12187/tunisie-tous-les-pecheurs-de-zarzis-ontdeja-secouru-des-migrants-en-mer-et-on-continuera.

26. "Tunisie - Racisme : pour les ressortissants subsahariens, 'trop c'est trop !'" [En ligne], Le Point.fr, 26 décembre 2018, https://www.lepoint.fr/afrique/tunisie-racisme-pour-lesressortissants-subsahariens-trop-c-est-trop-26-12-2018-2281900_3826.php\#.

27. BEN SEDRINE, Saïd, Défis à relever pour un accueil décent de la migration subsaharienne en Tunisie, op. cit. 
largement la représentation binaire d'un «avant » et d'un «après Ben Ali ». Comme nous le mentionnions précédemment, en matière migratoire, les éléments de continuité par rapport à la période autoritaire se combinent à des tendances à la rupture, confortant une dynamique politique paradoxale ${ }^{28}$.

Il est indéniable que depuis 20II, la Tunisie a connu des avancées législatives significatives concernant la protection des migrants, des réfugiés et des personnes vulnérables. La nouvelle Constitution tunisienne du 26 janvier 20I4, adoptée à la quasi unanimité des constituants, stipule que «le droit d'asile politique est garanti conformément aux dispositions de la loi, il est interdit de livrer les personnes qui bénéficient de l'asile politique $»^{29}$. C'est une première dans l'histoire de la Tunisie contemporaine et aussi du monde arabe. L'adoption de cet article doit beaucoup aux mobilisations des nouveaux parlementaires et des militants des droits de l'homme, anciens animateurs de la dissidence sous le régime autoritaire de Ben Ali, qui ont parfois vécu eux-mêmes le bannissement et l'exil politique en Europe ou dans le reste du monde ${ }^{30}$. C'est dans cet état esprit, afin de marquer une rupture nette avec les pratiques arbitraires de la dictature benaliste, que les constituants ont aussi adopté l'article 25, selon lequel «Aucun citoyen ne peut être déchu de la nationalité tunisienne, ni être exilé ou extradé, ni empêché de revenir dans son pays $»^{3 \mathrm{I}}$. La nouvelle Constitution consacre par ailleurs des droits civils, politiques, économiques et sociaux - notamment le droit d'association (article 35), la liberté de rassemblement et de manifestation publique (article 37), les libertés d'opinion, de pensée, d'expression, d'information et de publication (article 31), le droit à la santé (article 38 ), etc. ${ }^{32}$, - qui contribuent au processus de détabouisation de la parole publique sur des sujets autrefois censurés par la dictature comme le sort des jeunes harragas, l'exploitation économique des migrants, les discriminations et le racisme subis par les minorités immigrées, raciales et sexuelles : les porteurs de cause et les militants des droits des migrants se trouvent désormais protégés par le texte constitutionnel, même si cette

28. Sur la dynamique politique paradoxale de la «transition tunisienne », voir ALLAL, Amin ; GEISSER, Vincent, "Introduction: 'Good-Bye Ben Ali !'", Tunisie : une démocratisation audessus de tout soupçon?, op. cit., pp. 9-41.

29. Constitution tunisienne du 26 janvier 2014 : http://www.tunisie-constitution.org/fr/ar ticle-26-0.

30. Près d'un tiers des parlementaires élus à l'Assemblée nationale constituante (ANC), en octobre 2011, avait connu directement ou indirectement, sous la dictature, des situations de bannissement, d'exil ou de contrôle administratif limitant leur mobilité sur le territoire tunisien. Sur ce sujet, voir PEREZ, Déborah, "Faire la loi à I'Assemblée nationale constituante tunisienne. Recomposition et formation d'un nouveau personnel parlementaire tunisien", L'Année du Maghreb, n 14, 2016, pp. 187-204.

31. Constitution tunisienne du 26 janvier 2014: http://www.tunisie-constitution.org/fr/ar ticle-25-0.

32. BEN SEDRINE, Saïd, Défis à relever pour un accueil décent de la migration subsaharienne en Tunisie, op. cit. (voir pp. 57 et suivantes). 
protection est toute «théorique », entravée par la persistance de pratiques arbitraires héritées de l'ancien régime.

Sous l'effet des mobilisations de la société civile locale, mais aussi sous la pression des institutions internationales, les parlementaires tunisiens ont également adopté à l'unanimité, le 3 août 20I6, la loi organique relative à la prévention et la lutte contre la traite des personnes : « $\mathrm{La}$ présente loi vise à prévenir toutes formes d'exploitation auxquelles pourraient être exposées les personnes, notamment les femmes et les enfants, à lutter contre leur traite, en réprimer les auteurs et protéger et assister les victimes. Elle vise également à promouvoir la coordination nationale et la coopération internationale dans le domaine de la lutte contre la traite des personnes dans le cadre des conventions internationales, régionales et bilatérales ratifiées par la République tunisienne » (article I) $)^{33}$. La loi consacre d'ailleurs un article entier à la protection des personnes de nationalité étrangère résidant sur le territoire tunisien : "Est accordé à l'étranger qui peut être une victime de l'une des infractions de la traite des personnes prévues par la présente loi, le droit à une période de rétablissement et de réflexion qui peut atteindre un mois renouvelable une seule fois pour la même période. L'intéressé exerce ce droit sur sa demande en vue de pouvoir engager les procédures judiciaires et administratives. Il est interdit de le rapatrier au cours de cette période » (article 64) ${ }^{34}$.

Cela étant, de toutes les avancées législatives, c'est surtout la loi $\mathrm{n}^{\circ} \mathrm{II} / 20 \mathrm{I} 8$ sur l'élimination de toutes les formes de discriminations raciales qui a été la plus médiatisée, faisant l'objet de nombreux débats publics en Tunisie et à l'étranger. Si, en apparence, cette loi est d'initiative gouvernementale (discours du président de la République Béji Caïd Essebsi et du chef du gouvernement Youssef Chahed désignant le racisme comme étant contraire aux traditions d'hospitalité de la Tunisie) ${ }^{35}$, le texte est surtout le produit des mobilisations militantes, notamment des associations d'étudiants subsahariens et des organisations de Noirs tunisiens, dans un contexte social marqué par la montée des violences et des crimes racistes $^{36}$. Si le climat postrévolutionnaire a contribué à détabouiser la question du racisme anti-noir qui frappe autant des nationaux tunisiens (minorités noires, notamment dans le sud du pays) que des étrangers (étudiants et travailleurs d'Afrique de l'Ouest), elle a aussi libéré la «pa-

33. Loi organique $n^{\circ} 2016-61$ du 3 août 2016 , relative à la prévention et la lutte contre la traite des personnes: http://www.legislation.tn/sites/default/files/fraction-journal-officiel/ 2016/2016F/066/Tf2016611.pdf.

34. Ibidem.

35. "Tunisie: Youssef Chahed appelle à accélérer l'adoption d'une loi contre le racisme" [En ligne], Huffpostmaghreb.com, 26 décembre 2016, https://www.huffpostmaghreb.com/ 2016/12/26/youssef-chahed-racisme_n_13856594.html.

36. "Les étudiants africains sont-ils devenus 'indésirables' en Tunisie?" [En ligne], Espace Manager.com, 30 septembre 2013, https://www.france-terre-asile.org/actualites/ actualites/actualites-du-maghreb/les-etudiants-africains-sont-ils-devenusqindesirablesq-en-tunisie. 
role raciste » dans les médias, les enceintes sportives et chez certaines personnalités politiques tunisiennes. La Tunisie est aujourd'hui le premier pays arabe à avoir voté une telle loi qui prévoit des peines de prison et des amendes élevées pour toute personne ou tout groupe qui se livrerait à l'incitation à la haine, à des menaces racistes, à la diffusion et à l'apologie du racisme ${ }^{37}$. Il est vrai que les débats contradictoires entourant l'adoption de cette législation, inédite dans le monde arabe, ont suscité une forme de prise de conscience collective de l'existence d'un racisme anti-noir historiquement ancré. Car la société tunisienne a longtemps été dominée par un " discours prêt-à-dire », selon lequel la Tunisie serait un pays tolérant, musulman modéré et ouvert sur le monde ${ }^{38}$, immunisé contre les manifestations racistes comparables à celles de l'Europe. Bien sûr, des travaux sociologiques récents ont montré le contraire, en soulignant l'ancienneté et l'ampleur des préjugés anti-noirs dans les milieux populaires et les classes moyennes, mais aussi chez les élites tunisiennes ${ }^{39}$.

Ces avancées législatives et sociétales que connaît la Tunisie depuis la révolution de 2011 ne doivent pas pour autant faire oublier les formes de résiliences paternalistes, autoritaires et sécuritaires, les «poches d'immobilisme » et surtout les secteurs entiers de la société tunisienne qui continuent à échapper à l'application des règles de droit. Cette dernière donnée n'est pas négligeable quand on sait que la très grande majorité des travailleurs subsahariens résidant en Tunisie sont employés dans le secteur informel (agriculture, restauration/hôtellerie, bâtiment, aides à domicile, etc.), ne bénéficiant d'aucune protection sociale et juridique ${ }^{40}$. Mais il est vrai aussi, comme le montre le sociologue Mustapha Nasraoui, que le droit du travail tunisien constitue une machine à fabriquer de l'irrégularitét ${ }^{4}$, en ce sens que le Code du travail, très restrictif, induit une

37. BOBIN, Frédéric, "Loi pénalisant le racisme en Tunisie : une première victoire pour la minorité noire" [En ligne], Le Monde.fr, 11 octobre 2018, https://www.le monde.fr/afrique/article/2018/10/11/loi-penalisant-le-racisme-en-tunisie-une-premierevictoire-pour-la-minorite-noire_5368149_3212.html ; VERDIER, Marie, "La Tunisie s'attaque au racisme" [En ligne], La Croix.com, 12 octobre 2018, https://www.lacroix.com/Monde/Afrique/Tunisie-sattaque-racisme-2018-10-12-1200975628.

38. Ce discours idyllique sur la « légendaire tolérance tunisienne » s'appuie sur le fait historique que la Tunisie a été I'un des premiers États du monde à abolir l'esclavage, en 1846, sous le règne du Bey Ahmed $1^{\mathrm{er}}$.

39. ABDELHAMID, Maha, "De la libération de la parole raciste à l'émergence d'un mouvement contre le racisme anti-noir", op. cit.; MRAD-DALI, Inès, Identités multiples et multitudes d'histoires : les "Noirs tunisiens" de 1846 à aujourd'hui, thèse de doctorat en anthropologie sociale et ethnologie, Paris : EHESS, 2009.

40. Selon I'association Terre d'Asile, en 2016, sur les 10000 Africains subsahariens résidant en Tunisie (estimation), $75 \%$ étaient en situation irrégulière, ne disposant d'aucun titre de séjour valide, et se trouvant donc à la merci d'employeurs privés peu scrupuleux. Voir NASRAOUI, Mustapha, "Les travailleurs migrants subsahariens en Tunisie face aux restrictions législatives sur l'emploi des étrangers", Revue européenne des migrations internationales, vol. 33, $n^{\circ} 4$, 2017, pp. 159-178.

41. Souligné par nous. 
«préférence nationale » à peine déguisée. Or, ce Code n'a pas connu de révision approfondie depuis plus de trente ans et contribue donc à «irrégulariser ${ }^{4^{2}}$ de nombreux étrangers en provenance d'Afrique de l'Ouest, alors qu'il protège simultanément les étrangers européens et d'Amérique du Nord (coopérants, expatriés, entrepreneurs, hommes d'affaires, etc.) : "On ne peut toutefois passer sous silence un fait saillant dans l'attitude de l'État tunisien : la dissonance entre sa défense des droits des Tunisiens en situation irrégulière à l'étranger et son mutisme face aux droits des migrants subsahariens se trouvant dans la même situation sur son propre territoire, incohérence qui ne met pas à l'aise la majorité des citoyens »43. Ce processus d'irrégularisation par le droit s'appuie sur tout un arsenal législatif, notamment la loi $n^{\circ}$ 66-27 du 30 avril I966, qui, « complétée par la loi n 96-62 $d u$ I5 juillet 1996, introduit plusieurs restrictions concernant l'emploi des étrangers, notamment par son article 258-2: "Tout étranger qui veut exercer en Tunisie un travail salarié de quelque nature qu'il soit doit être muni d'un contrat de travail et d'une carte de séjour portant la mention 'autorisé à exercer un travail salarié en Tunisie”. Après avoir précisé la durée du contrat de travail (une année, renouvelable une seule fois), le même article ajoute : "Le recrutement d'étrangers ne peut être effectué lorsqu'il existe des compétences tunisiennes dans les spécialités concernées par le recrutement" ${ }^{44}$.

La conséquence dramatique du droit du travail tunisien, fondé sur un régime implicite de "préférence nationale », est de plonger chaque année des milliers de travailleurs subsahariens dans des situations d'irrégularité, alors que, précisément, leur vou le plus cher est d'obtenir des contrats formels. Comme le souligne l'économiste Saïd Ben Sedrine, «dans ce contexte, l'application rigide du principe d'opposabilité du marché du travail et le durcissement de la législation sur le séjour et l'emploi des étrangers segmentent le marché du travail en un marché régulier fermé aux travailleurs migrants africains et un marché informel où se réfugient les migrants irréguliers ${ }^{45}$. Au regard de ces observations, nous sommes bien en présence de politiques d'irrégularisation : la très grande majorité des migrants subsahariens sont donc assignés au secteur informel et aux traitements arbitraires et discriminatoires des employeurs tunisiens. L'exploitation économique et le racisme sont deux phénomènes étroitement liés, puisque les autorités publiques et les employeurs privés profitent très largement de cette situation d'irrégularité quasi structurelle pour imposer aux migrants subsahariens des conditions de travail inhumaines et pratiquer à

42. Sur la notion de «politiques d'irrégularisation» et le droit des étrangers, voir "Politiques d'irrégularisation" (dossier), Migrations Société, vol. 30, n 171, janvier-mars 2018, pp. 13-104.

43. NASRAOUI, Mustapha, "Les travailleurs migrants subsahariens en Tunisie face aux restrictions législatives sur l'emploi des étrangers", op. cit. (voir p. 173).

44. Ibidem (voir p. 161).

45. BEN SEDRINE, Saïd, Défis à relever pour un accueil décent de la migration subsaharienne en Tunisie, op.cit. (voir p. 7). 
leur égard toutes sortes d'actes d'humiliation : "Les immigrés de l'Afrique subsaharienne possèdent un ensemble de caractéristiques susceptibles de déclencher des actes discriminatoires et sont donc particulièrement exposés à l'inégalité de traitement. Les effets de plusieurs motifs de discrimination peuvent se combiner et, pour certaines personnes, rendre plus difficile l'exercice de leurs droits. C'est le cas des femmes africaines subsahariennes qui peuvent être victimes de discrimination raciale, mais aussi de discrimination fondée sur le genre $\gg^{46}$.

Ces situations d'injustice frappent particulièrement les femmes subsahariennes qui travaillent de plus en plus comme domestiques dans des habitations privées. Sur ce plan, notons que la Tunisie est marquée ces dernières années par une évolution " à la libanaise " ${ }^{47}$ ou «à la golfienne ${ }^{48}$, comparable aux traitements discriminatoires subis par les domestiques étrangères au Moyen-Orient et dans les pays du Golfe persique (Philippines, Éthiopiennes, Sri Lankaises, etc.), alors que cette forme d'exploitation était quasiment inexistante il y a encore quelques années. En effet, jusqu'aux années I970-I980, la Tunisie à l'instar du Maroc, était caractérisée par le système des « petites bonnes » provenant des régions déshéritées, notamment du Nord Ouest (le Kef, Jendouba, Bou Salem, Tabarka, etc.). Mais ce traitement inhumain des domestiques mineures a fait l'objet de nombreuses réprobations sociales, associatives et syndicales dans un pays qui proclamait ouvertement ses valeurs républicaines et son attachement à l'égalité hommes/femmes et où les mouvements féministes étaient plus influents que dans le reste du monde arabe. En conséquence, le phénomène s'était considérablement tari contrairement au Maroc où il persiste toujours en ce début de $\mathrm{XXI}^{\mathrm{e}}$ siècle $^{49}$ - au profit d'un système de femmes de ménage mineures ou adultes, que l'on qualifie de "couchantes "50 $^{5}$,'est-à-dire des employées féminines qui vivent et dorment au domicile de leur employeur. Si les conditions de ces femmes de ménage tunisiennes restent extrêmement difficiles et précaires, leurs papiers sont rarement confisqués et elles peuvent garder des contacts quotidiens avec leur famille (certaines même repartent le soir à leur domicile, une fois le travail terminé). Toutefois, l'évolution des formes de domesticité en Tunisie a évolué depuis la révo-

46. Ibidem (voir p. 41).

47. JUREIDINI, Ray, "L'échec de la protection de l'État: les domestiques étrangers au Liban", Revue européenne des migrations internationales, vol. 19, n³, 2003, pp. 95-127.

48. LAVERGNE, Marc, "Golfe arabo-persique : un système migratoire de plus en plus tourné vers I'Asie", Revue européenne des migrations internationales, vol. 19, n 3, 2003, pp. 229-241.

49. KADIRI, Ghalia, "Au Maroc, un décret controversé sur le travail des 'petites bonnes'" [En ligne], Le Monde.fr, 15 août 2017, https://www.lemonde.fr/afrique/article/2017/08/15/aumaroc-un-decret-controverse-sur-le-travail-des-petites-bonnes_5172545_3212.html.

50. SBOUAï, Sana, "Femme de ménage, bonne couchante : une vie dans l'ombre" [En ligne], Nawaat.org, 24 juillet 2012, https://nawaat.org/portail/2012/07/24/femme-de-menagebonne-couchante-une-vie-dans-lombre/. 
lution et ne touche plus exclusivement des femmes tunisiennes mais aussi des étrangères, en particulier des migrantes subsahariennes, tendant à rapprocher la situation locale des conditions d'exploitation féminine des pays comme le Liban, la Jordanie, Israël${ }^{51}$ et les pays du Golfe. Cette évolution peut apparaître choquante pour un pays qui se présente pourtant comme le modèle de "société démocratique " pour le monde arabe ${ }^{52}$ : "le processus de recrutement des femmes de ménage est très organisé. Il se fait d'abord depuis le pays d'origine par le biais de réseaux d'intermédiaires informels avec des pratiques illégales et abusives. Ces intermédiaires subsahariens repèrent les candidates à la migration, souvent soutenues dans ce projet par leur famille, puis ils organisent leur voyage en Tunisie (billet d'avion, bilan de santé, vaccin, et réservation d'hôtel). Les migrantes sont mises en contact avec d'autres intermédiaires en Tunisie chargés de les présenter à leurs employeurs. Le service de ces intermédiaires est payant à la fois pour les travailleuses migrantes et les employeurs. Les frais sont réglés en espèces avant le départ des migrantes. Les employeurs payent les services de l'intermédiaire local qui sont parfois prélevés sur le salaire de l'employée de maison africaine [...] »53.

Ces situations socioprofessionnelles peu enviables n'épargnent pas non plus les hommes subsahariens (selon les enquêtes récentes, $75 \%$ d'entre eux travailleraient aujourd'hui dans le secteur informel). On les recense majoritairement dans les sous-secteurs de l'agriculture, du bâtiment, des travaux publics, de la restauration, du gardiennage, etc. Certains parmi eux sont venus en Tunisie par les réseaux de trafics des « jeunes footballeurs », mais la majorité d'entre eux n'est jamais parvenue à décrocher le moindre contrat auprès d'un club, se retrouvant dans des situations d'exploitation criantes : bas salaires, durée du travail dépassant largement la limite horaire légale, absence de protection sociale et juridique et racisme professionnel de type paternaliste de la part des employeurs tunisiens qui les considèrent toujours comme des abid (esclaves), des wsif (domestiques), des kahlouche (négros, nègres), ou des Bila ${ }^{54}$, etc. ${ }^{55}$ Un rapport de 2017 réalisé par des experts pour le compte des ONG Terre d'Asile Tunisie et Médecins du monde souligne d'ailleurs l'état de souf-

51. GEISSER, Vincent, "La Terre promise... mais pas pour tout le monde. De la fiction à la réalité de la xénophobie anti-africaine en Israël", Migrations Société, vol. 24, n 141-142, mai-août 2012, pp. 3-12.

52. Sur ce phénomène de la nouvelle domesticité subsaharienne en Tunisie, voir NASRAOUI, Mustapha, "Les travailleurs migrants subsahariens en Tunisie face aux restrictions législatives sur l'emploi des étrangers", op. cit. (voir pp. 159 et suivantes).

53. BEN SEDRINE, Saïd, Défis à relever pour un accueil décent de la migration subsaharienne en Tunisie, op. cit. (voir p. 46).

54. Bilal était le prénom d'un compagnon noir du Prophète Mohamed qui était aussi son muezzin. Par extension, il est devenu une manière, plus ou moins stigmatisante, de désigner les « musulmans noirs ».

55. Sur le vocabulaire du racisme anti-noir en Tunisie, voir ABDELHAMID, Maha, "De la libération de la parole raciste à l'émergence d'un mouvement contre le racisme anti-noir", op. cit. (voir p. 351). 
france psychologique dans lequel se trouvent la majorité des migrants subsahariens en Tunisie : "une proportion importante de migrants subsahariens interrogés ont été victimes d'agressions de la part de Tunisiens : $69 \%$ ont été victimes de vol avec violence, agression sexuelle, lapidation ou autres. $69 \%$ des Subsahariens ont également déclaré se sentir en insécurité physique en Tunisie $»^{56}$.

Au regard de ce tour d'horizon de la situation des migrants subsahariens dans la société tunisienne post-20II, il apparaît clairement que les politiques étatiques et, d'une manière générale, les dispositifs administratifs déployés pour le contrôle des migrations, n'ont pas rompu avec le paradigme dominant de la période autoritaire. Ce constat lucide est partagé par la plupart des acteurs associatifs ${ }^{57}$, des experts et des chercheurs sur la question migratoire : "Plusieurs années après les soulèvements populaires de 20II, cette même hiérarchie de priorités, fondée sur un paradigme sécuritaire, continue d'orienter les choix des acteurs politiques et institutionnels en Tunisie ${ }{ }^{5}$, relève Jean-Pierre Cassarino. Pour preuve, alors que l'asile est désormais un droit garanti par la nouvelle Constitution tunisienne du 26 janvier 20I4, aucune loi sur l'asile n'a encore été votée par le Parlement, les responsables politiques et gouvernementaux craignant qu'elle n'encourage les migrants subsahariens à transiter ou, pire, à s'installer durablement sur le territoire tunisien: «la ratification de la Convention de Genève sur l'asile (195I) par la Tunisie n'a en effet pas été suivie par sa transcription dans le droit interne. Le droit d'asile n'est de ce fait pas reconnu, ni dans la législation, ni dans la pratique de l'État tunisien ${ }^{59}$. Plus que jamais, la combinatoire sécuritaire lutte contre les migrations « clandestines»/lutte contre le terrorisme islamique est devenue, pour les États européens et les bailleurs de fonds, le principal critère d'évaluation de la «bonne gouvernance tunisienne », comme il l'était déjà d'ailleurs à l'époque de la dictature de Ben Ali. Toutefois, cette continuité sécuritaire ne relève pas exclusivement d'une résilience autoritaire, c'est-à-dire des legs paternalistes et répressifs hérités de l'ancien régime. De ce point de vue, la reconduction de la gestion sécuritaire des migrations apparaît

56. CLAESSENS, Élisa; LAROUSSI, Dalel, La santé mentale des migrants en Tunisie. Quels facteurs de vulnérabilité et quels soins proposer?, Tunis: Terre d'Asile Tunisie/Médecins du monde, 2017, 68 p. (voir p. 34).

57. HAON, Nicanor, "Tunisie : pas de printemps pour les migrants", Plein droit, vol. 94, $n^{\circ} 3$, 2012, pp. 31-35.

58. CASSARINO, Jean-Pierre, "Le gouvernement des migrations en Tunisie : vers un nouveau paradigme?", op.cit. (voir p. 297).

59. NASRAOUI, Mustapha. «Les travailleurs migrants subsahariens en Tunisie face aux restrictions législatives sur l'emploi des étrangers », op.cit. (voir p. 160). 
autant comme un héritage de l'ancien système que l'un des acquis profondément ambivalent et faussement «démocratique » du nouveau régime, largement encouragé par l'Union européenne et les principaux partenaires de la Tunisie: considéré du temps de Ben Ali comme le « modèle arabe et africain » en matière de lutte contre le terrorisme et l'immigration "clandestine », la Tunisie démocratique espère retrouver sa place dans la classe des élèves méritants. Mais n'oublions pas qu'aux yeux des décideurs européens, la «bonne démocratie arabe » est celle qui tient d'une main de fer son peuple et ses migrants, quitte à fermer les yeux sur la répression des mouvements sociaux et des courants contestataires.

Marseille, le I3 septembre 2019 